\title{
RESEARCH
}

\section{Evaluating the Pre-Multistate Pharmacy Jurisprudence Exam and Other Factors Associated with Performance on the Multistate Pharmacy Jurisprudence Exam}

\author{
Dawn E. Havrda, PharmD, Elizabeth A. Hall, PharmD, Christina A. Spivey, PhD, Lauren T. Biliter, Rachel E. \\ Barenie, PharmD, JD, MPH, Marie A. Chisholm-Burns, PharmD, PhD, MBA, MPH \\ University of Tennessee Health Science Center, College of Pharmacy, Memphis, Tennessee
}

Corresponding Author: Dawn Havrda, University of Tennessee Health Science Center, College of Pharmacy, 881 Madison Ave., Memphis, TN 38163. Tel: 901-448-1841. Email: dhavrda@uthsc.edu

Submitted June 15, 2021; accepted October 15, 2021; ePublished October 2021

\begin{abstract}
Objective. To evaluate the relationship between the Pre-Multistate Pharmacy Jurisprudence Examination (MPJE) and MPJE among pharmacy graduates and determine the influence of demographic, pre-pharmacy, and pharmacy school factors on MPJE outcomes.
\end{abstract}

Methods. A retrospective review of pharmacy graduates' ( $n=156)$ MPJE scores, Pre-MPJE scores, demographics, prepharmacy academic performance factors, and pharmacy school academic performance factors was performed. Bivariate and correlational analyses were conducted along with multiple linear regression modules to determine the influence of variables on the MPJE total scaled score.

Results. A total of 136 graduates were included with the majority being female (59\%) and non-Hispanic White students (75\%). The Pre-MPJE was not significantly correlated with the MPJE first attempt pass/fail outcome or total scaled score. Younger age at graduation, higher pharmacy law course grade, PCOA total and content area 1-4 scaled scores, and final pharmacy GPA were correlated to passing the MPJE. MPJE total scaled score was correlated to higher pre-pharmacy GPA, pharmacy law course grade, PCOA total and content area 1-4 scaled scores, and final pharmacy GPA. However, regression models found the greatest variance in MPJE total scaled score was contributed by the pharmacy law course grade. PCOA total scaled score contributed to some variance for all MPJE takers, but only pharmacy law course grade significantly influenced in-state MPJE total scaled score.

Conclusion. The findings did not show pre-MPJE was a predictor for passing or total scaled score for the MPJE. Pharmacy law course performance was the most important determinant of MPJE total scaled score.

Keywords: MPJE, pre-MPJE, student characteristics

\section{INTRODUCTION}

First-time board pass rates are key quality metrics for Doctor of Pharmacy (PharmD) programs in the United States. The Accreditation Council for Pharmacy Education (ACPE) requires accredited programs to publish current North American Pharmacist Licensure Examination (NAPLEX) pass rates for the graduating class. ${ }^{1}$ However, becoming a licensed pharmacist often also requires passing the Multistate Pharmacy Jurisprudence Examination (MPJE) specific to the jurisdiction the graduate plans to practice in.

In 2020, the National Association of Boards of Pharmacy (NABP), which administers the MPJE with state boards of pharmacy, offered a Pre-MPJE for the first time. The Pre-MPJE is a state-specific practice exam for a candidate to become familiar with the MPJE question format and testing process. The Pre-MPJE consists of 40 questions from prior MPJE exams within 50 minutes. The candidate receives a scaled score ranging from 0 to $100 .{ }^{2}$ Comparatively, the MPJE is a 2.5-hour adaptive exam with 120 questions of which 100 questions are used to calculate the graduate's results. Before 2021, the minimum passing scaled score was 75 for the MPJE with a range from 0 to $100 .^{3}$

Similarly, the NABP offers the Pre-NAPLEX for self-assessment and familiarization with the NAPLEX exam format. In 2006, Peak and colleagues surveyed graduates regarding tools used to prepare for NAPLEX and MPJE. The authors found the Pre-NAPLEX was the most recommended and representative preparatory tool, used by $26.7 \%$ of graduates. ${ }^{4}$ Chisholm-Burns and colleagues examined the relationship of the Pre-NAPLEX and NAPLEX. Results indicated Pre-NAPLEX scores along with final pharmacy GPA predicted a positive correlation with NAPLEX total scaled scores. ${ }^{5}$ No prior studies have evaluated if the Pre-MPJE predicts MPJE total scaled scores. Therefore, we aimed to evaluate whether Pre-MPJE scores were correlated to Tennessee MPJE or out-of-state MPJE scores or pass rates and the influence of demographics and pre-pharmacy and pharmacy school performance on MPJE outcomes. 


\section{METHODS}

This was a retrospective study of the graduating PharmD class of $2020(n=156)$ at the University of Tennessee Health Science Center (UTHSC) College of Pharmacy (COP). The Pre-MPJE was administered within a licensure preparatory course. The COP provided all graduates a voucher code for the Tennessee Pre-MPJE to take between April 17-21, 2020. No Pre-MPJE minimum score was established to pass the COP's course, and graduates submitted a screenshot of their score. This Pre-MPJE testing period occurred approximately one week before the required half-day COP-sponsored Tennessee law review and was intended as a self-assessment for the MPJE.

Students were asked to sign a release allowing NABP to provide the COP with their MPJE score. First-time pass rates and total scaled scores for the MPJE were collected for students who took the MPJE between May and December 2020. Data retrieved from student records included: demographics (age at graduation, gender, race/ethnicity); Pharmacy College Admission Test (PCAT) composite score; pre-pharmacy GPA; pharmacy law course final grade (presented on a GPA-based scale: $\mathrm{A}=4.0, \mathrm{~A}-=3.67, \mathrm{~B}+=3.33, \mathrm{~B}=3.0, \mathrm{~B}-=2.67, \mathrm{C}+=2.33, \mathrm{C}=2.0, \mathrm{C}-=1.67, \mathrm{D}=1.0$ and $\mathrm{F}=0$ ); final GPA at graduation (final pharmacy GPA); Pharmacy Curriculum Outcomes Assessment (PCOA) total scaled score and scaled scores of four content areas (Area 1: basic biomedical sciences; Area 2: pharmaceutical sciences; Area 3: social/behavioral/administrative sciences; and Area 4: clinical sciences ${ }^{6}$; and history of academic difficulty during the PharmD program. Academic difficulty was defined as earning at least one grade below a C- or a "no pass" in any course. Students who did not take the MPJE during the study period or did not consent to release their MPJE scores to the COP were excluded. The UTHSC Institutional Review Board approved this study.

Data analyses were conducted using IBM SPSS Statistics 26.0 (Armonk, New York). Descriptive statistics and frequency counts were performed for demographic variables. Independent samples t-test (or Mann-Whitney U test when data were not normally distributed) and Chi-square analyses were conducted to compare class of 2020 graduates who have taken the MPJE to those who were excluded on the following variables: age, gender, race/ethnicity, PCAT composite score, pre-pharmacy GPA, final pharmacy law grade, PCOA total scaled score, PCOA content area scaled scores, history of academic difficulty, final pharmacy GPA, and Pre-MPJE score.

Among graduates who took the MPJE, Chi-square analysis was conducted to determine differences in proportion of students passing the MPJE on first attempt based on gender, race/ethnicity, and history of academic difficulty. An independent samples t-test was conducted to assess differences in Pre-MPJE score based on whether or not students passed the MPJE. Chi-square analysis was conducted to determine differences in proportion of students passing the MPJE on first attempt based on subgroups of those who took the Tennessee MPJE compared to those who took an out-of-state MPJE. Correlational analysis (Spearman rho and point biserial) was conducted to assess relationships among PCAT composite score, pre-pharmacy GPA, final pharmacy law grade, final pharmacy GPA, PCOA total scaled score, PCOA content area scaled scores, Pre-MPJE score, MPJE total scaled score, and MPJE pass/fail outcome. Cohen's conventions were used to interpret strength of correlations, with .1 indicating a small effect, at least .3 suggesting a medium effect, and at least .5 implying a large effect. ${ }^{7}$

In the primary analysis, a multiple linear regression was conducted to determine the influence of the following 10 variables on MPJE scaled score in the total sample: age, gender, race/ethnicity, PCAT composite score, pre-pharmacy GPA, final pharmacy law grade, PCOA total scaled score, history of academic difficulty, final pharmacy GPA, and PreMPJE score. Multiple logistic regression analysis was likewise conducted to determine the influence of the same aforementioned 10 variables on first attempt MPJE pass/fail outcome in the total sample. Variables were entered in one block simultaneously in the linear regression and logistic regression analyses. To determine the final model (regression equation), nonsignificant variables were removed from the model, and the analysis was repeated. A priori alpha level of .05 was set for regression model.

In secondary analysis, the multiple linear regression described above was replicated, with the exception that the PCOA total scaled score was replaced in the model by the PCOA content area scaled scores. In the secondary analyses, separate multiple linear regression models were tested for two subgroups: those who took the Tennessee MPJE and those who took an out-of-state MPJE. The same variables were included in each model as in the primary analysis. Variables were entered in the model in one block simultaneously. A priori alpha level was set at .05. A sample size calculation was conducted for the primary analysis, multiple linear regression with 10 predictor variables. Power was set at 0.80 and significance at .05 , with a medium effect type. Sample size needed to achieve power of 0.80 was 117 .

\section{RESULTS}

Student characteristics are presented in Table 1. Most were female (59\%) and non-Hispanic White (75\%) students. Of 156 graduates, $136(87.2 \%)$ took the MPJE during the study period; of the remaining 20 graduates, 13 did not take the MPJE and 7 did not consent to release their scores. Among those 136 graduates, 124 (91.2\%) passed the MPJE on 
the first attempt. Additionally, 101 of the 136 took the Tennessee MPJE, while the remaining 35 took an out-of-state MPJE. In comparing the study population who took the MPJE and those excluded, the latter group had a significantly lower pharmacy law course final grade $(p=.002)$ and final pharmacy GPA $(p=.001)$.

In the Chi-square analysis, there were no significant differences in the proportion of students who passed the MPJE on the first attempt based on gender $(p=.99)$, race/ethnicity $(p=.12)$, or history of academic difficulty $(p=.11)$. There was also no difference in the Tennessee MPJE $(90.1 \%)$ or out-of-state MPJE $(94.3 \% ; p=.45)$ pass rate. In the correlational analysis (Table 2), passing the MPJE was significantly associated $(p<.05)$ with age at graduation, pharmacy law course grade, PCOA total scaled score, PCOA content areas 1-4 scaled scores, final pharmacy GPA, and MPJE score. MPJE total scaled score was significantly associated $(p<.05)$ with pre-pharmacy GPA, pharmacy law course grade, PCOA total scaled score, PCOA content areas 1-4 scaled scores, and final pharmacy GPA. Pre-MPJE score was not significantly correlated with the MPJE total scaled score or passing the MPJE, nor did Pre-MPJE score significantly differ in an independent sample t-test based on whether or not students passed the MPJE ( $M=73.99, S D=4.74$ vs. $M=72.33, S D=5.47$, respectively; $p=.26)$.

In the primary multiple linear regression analysis, pharmacy law course grade and PCOA total scaled score were significantly associated with MPJE score (Table 3). The final model, which included only pharmacy law course grade and PCOA total scaled score, explained $30.3 \%$ (adjusted $\mathrm{R}^{2}=0.303$ ) of the total variance in MPJE total scaled score; specifically, the pharmacy law course grade explained $27.3 \%$ (adjusted $\mathrm{R}^{2}=0.273$ ) and PCOA total scaled score explained the remaining $2.5 \%$ (adjusted $\mathrm{R}^{2}=0.025$ ) of the variance. The final linear regression equation was:

MPJE Total Scaled Score $=66.12+2.38$ (Pharmacy Law Course Final Grade $)+0.02$ (PCOA total scaled score)

In the multiple logistic regression analysis, none of the variables entered into the model was a significant predictor of first attempt passing/failing the MPJE.

In the secondary multiple linear regression analysis in which PCOA total scaled score was replaced by PCOA content area scaled scores as independent variables, pharmacy law course grade and PCOA content area 4 scaled score were significantly associated with MPJE score (Table 4). The final model, which included only pharmacy law course grade and PCOA content area 4 scaled score, explained 31.3\% (adjusted $\mathrm{R}^{2}=0.313$ ) of the total variance in MPJE total scaled score; specifically, the pharmacy law course grade explained $27.3 \%$ (adjusted $\mathrm{R}^{2}=0.273$ ) and PCOA content area 4 scaled score explained the remaining $4 \%$ (adjusted $\mathrm{R}^{2}=0.04$ ) of the variance. The final linear regression equation was:

MPJE Total Scaled Score $=66.9+2.27$ (Pharmacy Law Course Final Grade $)+0.02($ PCOA Content Area 4 scaled score)

In the secondary multiple linear regression analysis of the Tennessee MPJE subgroup ( $\mathrm{n}=136)$, only pharmacy law course grade was significantly associated with MPJE total scaled score (Table 5). The final model, which included only pharmacy law course grade, explained $33.4 \%$ (adjusted $\mathrm{R}^{2}=0.334$ ) of the total variance in MPJE score. The final linear regression equation for the Tennessee MPJE subgroup was:

MPJE Total Scaled Score $=70.83+2.96$ (Pharmacy Law Course Final Grade)

In the secondary multiple linear regression analysis of the out-of-state MPJE subgroup, pharmacy law course grade and PCOA total scaled score were significantly associated with MPJE total scaled score (Table 5). The final model which included pharmacy law course grade and PCOA total scaled score explained $30.9 \%$ (adjusted $\mathrm{R}^{2}=0.309$ ) of the total variance in MPJE total scaled score; specifically, pharmacy law course grade explained $24 \%$ (adjusted $\mathrm{R}^{2}=0.24$ ) and PCOA total scaled score explained the remaining $6.9 \%$ (adjusted $\mathrm{R}^{2}=0.069$ ) of the variance. The final linear regression equation for the out-of-state MPJE subgroup was:

MPJE Total Scaled Score $=55.03+2.07$ (Pharmacy Law Course Final Grade) + 0.05(PCOA total scaled score)

\section{DISCUSSION}

This is the first study to examine the relationship between the Pre-MPJE and MPJE performance. Previous research found the pre-NAPLEX was a predictor of NAPLEX score, but our analysis did not observe this relationship between Pre-MPJE and MPJE performance. ${ }^{5}$ Higher final pharmacy GPA, PCOA total and content area 1-4 scaled scores, and pharmacy law course grade had a significant correlation to passing the MPJE and total scaled score; younger age at graduation was also significantly correlated to passing the MPJE. In regression models, pharmacy law course grade accounted for much of the variance of the MPJE total scaled score, signaling it was the most important determinant of MPJE score. The PCOA total and content area 4 scaled scores were also found to influence MPJE outcomes, but not as much as the pharmacy law course grade.

The MPJE assesses pharmacy law core competencies involving both federal and state laws and regulations. ${ }^{8}$ In contrast to the NAPLEX, few courses in PharmD curricula comprehensively prepare graduates for the MPJE beyond the program's pharmacy law course. The Pre-MPJE is state specific and includes questions from previous MPJE exams. ${ }^{2}$ 
Theoretically, it could become a popular preparatory tool for the MPJE, similar to the Pre-NAPLEX. ${ }^{4}$ A significant relationship was not found between Pre-MPJE score and MPJE total scaled score or passing/failing on the first attempt in our analysis. An explanation for the lack of a statistically significant relationship between the Pre-MPJE and MPJE may be the sampling and number of questions on the Pre-MPJE. The Pre-MPJE contains 40 questions, or 33\% of the questions on the MPJE, whereas the Pre-NAPLEX contains 100 questions. ${ }^{2,3,9}$ More questions may be needed on the Pre-MPJE for a better pre-assessment. Additionally, some students may not have taken the self-assessment as intended without incentives nor a specific score requirement. Lastly, there is a lack of transparency regarding how questions are chosen for the PreMPJE. Using prior questions or testing new questions may not represent current MPJE content. Further analysis of the relationship, or lack thereof, between the Pre-MPJE and MPJE is needed given the cost to the test-taker for the Pre-MPJE.

Research remains limited on predictors of MPJE performance. This study found the pharmacy law course grade was the best indicator of passing the MPJE and MPJE score. When we examined the impact of pharmacy law grade on the Tennessee MPJE compared to out-of-state MPJE total scaled score, the course grade remained significantly associated. Similarly, Mospan and colleagues identified significant correlations between the North Carolina MPJE scores and undergraduate GPA, academic performance (including pharmacy law course grade), and PCOA performance. ${ }^{10}$ However, overall GPA was the strongest correlate, whereas we found pharmacy law course grade was a better predictor. While final pharmacy GPA was associated with MPJE score, final pharmacy GPA was not a significant factor in regression models when controlling for other independent factors. Additionally, three published conference abstracts found an association with final pharmacy GPA and MPJE score; however, pharmacy law course performance was not evaluated. ${ }^{11-13}$ Mospan and colleagues note their two-credit-hour pharmacy law course was delivered in fall of the second professional year and predominantly taught by a practicing pharmacist. ${ }^{10,14}$ At UTHSC COP, the pharmacy law course is three credit-hours, delivered in fall of the third professional year, and taught by an attorney. The course covers federal and state law and aligns with the MPJE Core Competencies. ${ }^{8}$ It is unknown if the timing, content, delivery, or instructor of pharmacy law courses impacts MPJE pass rates. A recent review by Rosenberg and colleagues found heterogeneity in law content of 49 PharmD programs, including timing within the curriculum, credit-hours, and type of instructor, but did not evaluate MPJE pass rates. ${ }^{15}$ Differences in pharmacy law courses may account for variations between the studies.

PCOA overall scaled score contributed to some of the variance for the overall MPJE scaled score and out-of-state MPJE scaled score, but not the Tennessee score. Mospan and colleagues found a positive correlation between MPJE score and PCOA overall score, but did not examine differences between their state versus out-of-state MPJE performance. ${ }^{10}$ Various studies have found PCOA total scores and area scores are correlated to NAPLEX total scaled score, but this is the first study to discover the association with MPJE total scaled score in regression models. ${ }^{16-19}$ A positive correlation was found with MPJE total scaled score and pass/fail outcome with the PCOA content areas 1-4 scaled scores; however, only content area 4 (clinical sciences) score was found to significantly contribute to the variance in the overall MPJE score in the regression model. Mospan and colleagues found significant correlations with the North Carolina MPJE score and PCOA content area 3 and law score. ${ }^{10}$ We did not examine the PCOA law score since it comprised only 5 questions of the exam. PCOA content area 4 comprises $35 \%$ of the exam and includes application of clinical sciences which may be more similar to the MPJE question format. ${ }^{6}$

In our regression analysis, PCOA total scaled score did not significantly influence Tennessee MPJE scores, but it was significant in the out-of-state MPJE scores model. The lack of significance of PCOA total scaled score on Tennessee MPJE score highlights the importance of the pharmacy law course in the PharmD curriculum, particularly for students planning to be licensed in the same state. For students testing outside of the state of their PharmD program, both pharmacy law course and overall PCOA performance influenced MPJE performance, demonstrating the effects of a specialized law course and the ability to apply and recall material taught throughout the curriculum.

Unlike past evaluations that assessed predictors of MPJE scores, we also examined predictors of passing the MPJE. In January 2021, the NABP announced NAPLEX and MPJE results would be reported as pass or fail only, with total scaled score no longer being provided ${ }^{20}$ As a result, colleges/schools will no longer be able to evaluate programspecific predictors related to scaled scores and will need to focus on pass/fail outcomes. Our study found a correlation with passing the MPJE to final pharmacy GPA, pharmacy law course grade, and PCOA total and content area 1-4 scaled scores. However, no significant findings were found in logistic regression analysis.

This study is not without limitations. First, generalizability may be limited as only students at one state public college of pharmacy were included. Most students took the Tennessee MPJE. Given MPJE varies per jurisdiction, programs should examine benefits of the pre-MPJE for their state. Second, only one year of data was available to evaluate the Pre-MPJE, which limits the sample size. However, the Pre-MPJE was first available in 2020, and MPJE total scaled scores will not be available to programs beginning January 2021. Third, MPJE results were not available for 20 graduates. 
Lastly, the pre-MPJE and MPJE were taken during a global pandemic, and it is unclear how that may have influenced the findings.

Future directions for research include analyzing factors that predict MPJE pass/fail outcome with the change in reporting of MPJE results, and the impact of pharmacy law course timing and instructor credentials on MPJE outcomes.

\section{CONCLUSION}

Based on experience at one public college of pharmacy, the Pre-MPJE does not predict performance or passing the MPJE. Pharmacy law course performance was the most significant contributor to predicting MPJE performance, both in-state and out-of-state. Given the impact of pharmacy law course grade on MPJE total scaled score, targeting students who had academic difficulty in the course for MPJE preparation may improve student performance.

\section{ACKNOWLEDGEMENTS AND DISCLOSURES}

The authors would like to thank Carol Schwab, JD, Professor, University of Tennessee Health Science Center for her time and dedication to education and the COP.

Marie Chisholm-Burns serves on the board of directors for the Accreditation Council of Pharmacy Education (ACPE). This manuscript does not represent ACPE or the boards' opinions or views.

\section{REFERENCES}

1. Accreditation Council for Pharmacy Education. Accreditation Standards and Key Elements for the Professional Program in Pharmacy Leading to the Doctor of Pharmacy Degree ("Standards 2016"). https://www.acpeaccredit.org/pdf/Standards2016FINAL.pdf. Accessed April 29, 2021.

2. National Association of Boards of Pharmacy. MPJE Practice exam. https://nabp.pharmacy/programs/examinations/mpje/practice-exam/. Accessed June 14, 2021.

3. National Association of Boards of Pharmacy. NAPLEX and MPJE 2020 Candidate Application Bulletin. 2020.

4. Peak AS, Sheehan AH, Arnett S. Perceived utility of pharmacy licensure examination preparation tools. Am J Pharm Educ. 2006;70(2):Article 25.

5. Chisholm-Burns MA, Spivey CA, Byrd DC, McDonough SLK, Phelps SJ. Examining the association between the NAPLEX, pre-NAPLEX, and pre- and post-admission factors. Am J Pharm Educ. 2017;81(5):Article 86.

6. National Association of Boards of Pharmacy. PCOA content areas. https://nabp.pharmacy/programs/examinations/pcoa/content-areas/. Published 2020. Accessed September 30, 2021.

7. Cohen J. Statistical Power Analysis for the Behavioral Sciences. 2nd ed. New York: Lawrence Erlbaum; 1988.

8. National Association of Boards of Pharmacy. MPJE competency statements. https://nabp.pharmacy/programs/examinations/mpje/competency-statements/. Published 2020. Accessed September 30, 2021.

9. National Association of Boards of Pharmacy. NAPLEX Practice exam. https://nabp.pharmacy/programs/examinations/naplex/practice-exam/. Accessed June 14, 2021.

10. Mospan G, Gillette C, Mospan CM. Predictors of performance on the North Carolina Multistate Pharmacy Jurisprudence Examination. Curr Pharm Teach Learn. 2020;12(1):35-40.

11. Lebovitz L, Shaya FT, Chirikov VV, et al. Admissions and other data as NAPLEX and MPJE performance determinants [abstract]. Am J Pharm Educ. 2014;78(5):Article 111.

12. Shaya FT, Chirikov VV, Lebovitz L, Schlesselman LS, Buring SM. The well-rounded applicant: admissions and other data as NAPLEX and MPJE performance determinants [abstract]. Am J Pharm Educ. 2015;79(5):Article S4.

13. Shaya F, Lebovitz L, Schlesselman LS, Buring SM. Three schools, five years of data: an update on NAPLEX and MPJE performance determinants [abstract]. Am J Pharm Educ. 2016;80(5):Article S2.

14. Wingate School of Pharmacy website. Academics - Courses. https://www.wingate.edu/academics/graduate/pharmacy/wusop-academics/course-list. Accessed June 14, 2021.

15. Rosenberg E, Albert EL, Mospan G, Panther S, Ruble J, Stein RL. A pilot study regarding pharmacy law education across doctor of pharmacy programs. Am J Pharm Educ. 2020;84(2):Article 7171.

16. Naughton CA, Friesner DL. Correlation of P3 PCOA scores with future NAPLEX scores. Curr Pharm Teach Learn. 2014;6(6):877-883.

17. Souza JM, Hutchinson DJ , Lenhard LM. Pharmacy Curriculum Outcomes Assessment (PCOA) as predictor of performance on NAPLEX [abstract]. Am J Pharm Educ. 2015;79(5):Article S4. 
18. Rudolph MJ, Gortney JS, Maerten-Rivera JL, et al. A study of the relationship between PCOA and NAPLEX using a multi-institutional sample. Am J Pharm Educ. 2019;83(2):Article 6867.

19. Daugherty KK, Malcom DR. Assessing the relationship between didactic performance and standardized examination scores in pharmacy students. Am J Pharm Educ. 2020;84(8):Article 847712.

20. National Association of Boards of Pharmacy. NAPLEX and MPJE Exam Results Announcement. 2021. https://nabp.pharmacy/news/news-releases/naplex-and-mpje-exam-results-announcement/. Accessed June 4, 2021. 
Table 1. Comparison of Demographics, Pre-Pharmacy Performance Indicators, and Pharmacy School Performance Indicators, among Class of 2020 Graduates $(n=156)$ Who Have and Have Not ${ }^{\mathrm{a}}$ Taken the MPJE, and First-attempt MPJE Outcomes

\begin{tabular}{|c|c|c|c|c|}
\hline & $\begin{array}{c}\text { Total Class } \\
n=156\end{array}$ & $\begin{array}{l}\text { Have Taken } \\
\text { MPJE } n=136\end{array}$ & $\begin{array}{c}\text { No MPJE Score }{ }^{\mathrm{a}} \\
\mathbf{n}=\mathbf{2 0}\end{array}$ & $p$ value \\
\hline Age at graduation, Mean (SD) & $28.1(3.45)$ & $28(3.45)$ & $28.7(3.51)$ & .22 \\
\hline Gender, n (\%) & & & & .56 \\
\hline Female & $92(59)$ & $79(58.1)$ & $13(65)$ & \\
\hline Male & $64(41)$ & $57(41.9)$ & $7(35)$ & \\
\hline Race/ethnicity, n (\%) & & & & .27 \\
\hline Non-Hispanic White & $117(75)$ & $104(76.5)$ & $13(65)$ & \\
\hline Minority & $39(25)$ & $32(23.5)$ & $7(35)$ & \\
\hline $\begin{array}{l}\text { PCAT composite score, Mean } \\
\text { (SD) }\end{array}$ & $68.6(21.14)$ & $69.5(20.62)$ & $62.5(24.12)$ & .22 \\
\hline $\begin{array}{l}\text { Pre-pharmacy GPA, Mean (SD) } \\
\text { Academic difficulty during } \\
\text { PharmD program } n(\%)\end{array}$ & $3.38(0.4)$ & $3.41(0.4)$ & $3.21(0.4)$ & .06 \\
\hline $\begin{array}{l}\text { PharmD program, n }(\%) \\
\text { No } \\
\text { Yes }^{b}\end{array}$ & $\begin{array}{l}128(82.1) \\
28(17.9)\end{array}$ & $\begin{array}{l}113(83.1) \\
23(16.9)\end{array}$ & $\begin{array}{l}15(75) \\
5(25)\end{array}$ & .38 \\
\hline $\begin{array}{l}\text { Pharmacy law course final } \\
\operatorname{grade}^{c}, \text { Mean }(\mathrm{SD})\end{array}$ & $2.96(0.7)$ & $3.02(0.7)$ & $2.52(0.56)$ & .002 \\
\hline $\begin{array}{l}\text { PCOA total scaled score, Mean } \\
\text { (SD) }\end{array}$ & $374.1(43.3)$ & $376.3(43.5)$ & $359.7(40.4)$ & .11 \\
\hline $\begin{array}{l}\text { PCOA Content Area } 1^{\mathrm{d}} \text { scaled } \\
\text { score, Mean }(\mathrm{SD}) \\
\text { PCOA Content Area } 2^{\mathrm{d}} \text { scaled }\end{array}$ & $355.1(72.9)$ & $353.44(72.2)$ & $366.5(78.3)$ & .46 \\
\hline $\begin{array}{l}\text { score, Mean (SD) } \\
\text { PCOA Content Area } 3^{\mathrm{d}} \text { scaled }\end{array}$ & $371.8(48.9)$ & $373.3(49.8)$ & $361.4(42.3)$ & .31 \\
\hline $\begin{array}{l}\text { score, Mean (SD) } \\
\text { PCOA Content Area } 4{ }^{\mathrm{d}} \text { scaled }\end{array}$ & $370(53.7)$ & $373.8(51.4)$ & $344.3(62.8)$ & .02 \\
\hline $\begin{array}{l}\text { score, Mean (SD) } \\
\text { Final pharmacy GPA, Mean }\end{array}$ & $387.3(55.5)$ & $390.2(55.9)$ & $367.7(49.8)$ & .09 \\
\hline$(\mathrm{SD})$ & $3.41(0.31)$ & $3.44(0.3)$ & $3.19(0.28)$ & .001 \\
\hline Pre-MPJE score, Mean (SD) & $73.4(5.09)$ & $73.8(4.81)$ & $70.6(6.18)$ & .01 \\
\hline $\begin{array}{l}\text { MPJE total scaled score, Mean } \\
\text { (SD) } \\
\text { MPJE first-attempt outcome, } n \\
(\%)\end{array}$ & & $80(3.78)$ & & \\
\hline $\begin{array}{l}\text { Pass } \\
\text { Did not pass }\end{array}$ & & $\begin{array}{c}124(91.2) \\
12(8.8)\end{array}$ & & \\
\hline
\end{tabular}

GPA, grade point average; MPJE, Multistate Pharmacy Jurisprudence Examination; PCAT, Pharmacy College Admission Test; PharmD, Doctor of Pharmacy degree; PCOA, Pharmacy Curriculum Outcomes Assessment; SD, standard deviation

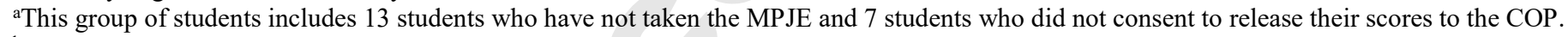
${ }^{b}$ Academic Difficulty refers to being placed on academic probation or having to appear before the Academic Standing and Promotion Review Committee at any point during the PharmD program.

cPharmacy Law Course Final grade presented on GPA scale where $\mathrm{A}=4.0, \mathrm{~A}-=3.67, \mathrm{~B}+=3.33, \mathrm{~B}=3.0, \mathrm{~B}-=2.67, \mathrm{C}+=2.33, \mathrm{C}=2.0, \mathrm{C}-=1.67, \mathrm{D}=1.0$, and $\mathrm{F}=0$.

${ }^{\mathrm{d}}$ The four PCOA content areas are basic biomedical sciences (Area 1), pharmaceutical sciences (Area 2), social/behavioral/administrative sciences (Area 3), and clinical sciences (Area 4). 
Table 2. Spearman Rho Correlations between Pre-Pharmacy Performance Indicators, Pharmacy School Performance Indicators, and First-attempt MPJE Outcomes $(n=136)$

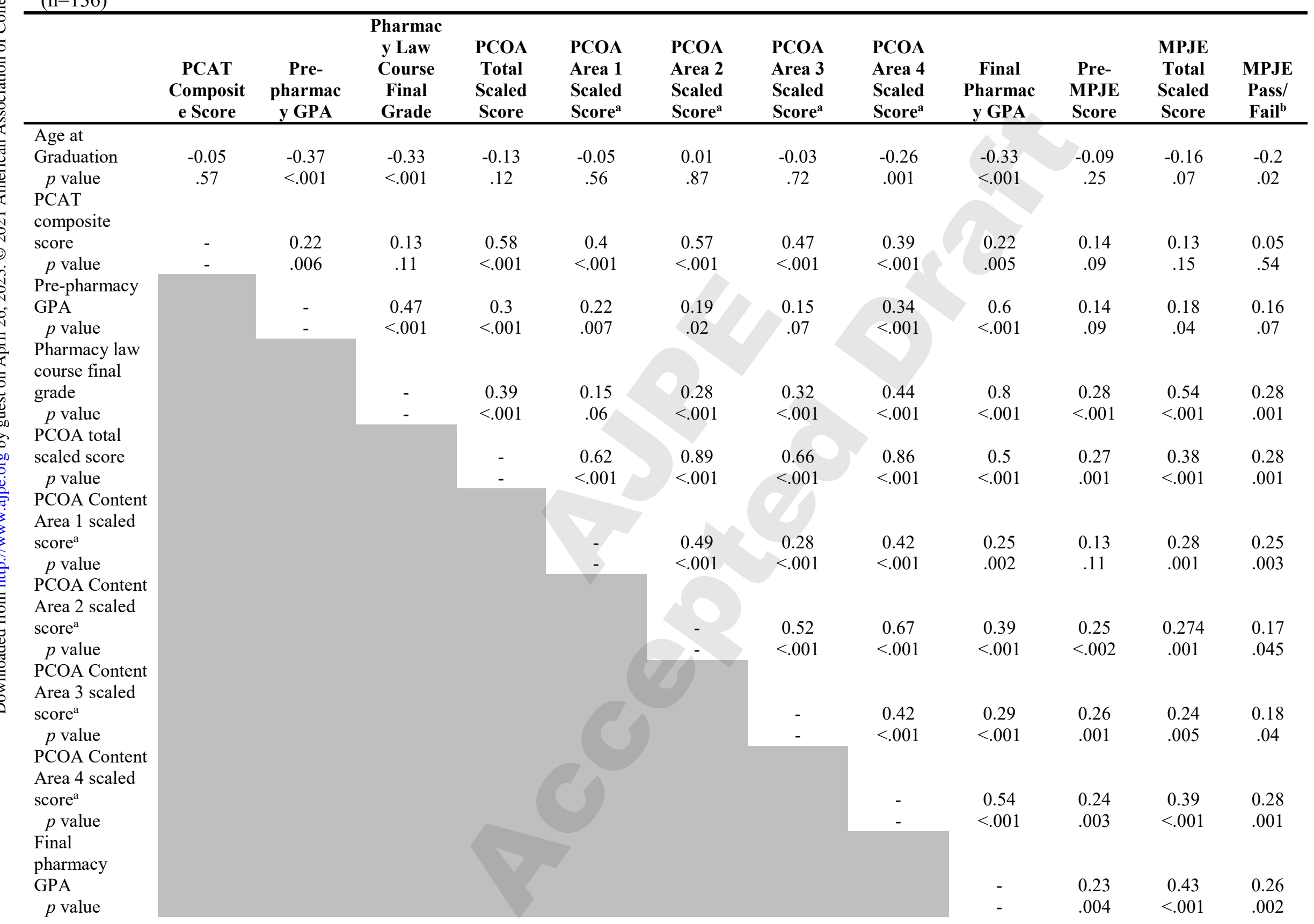




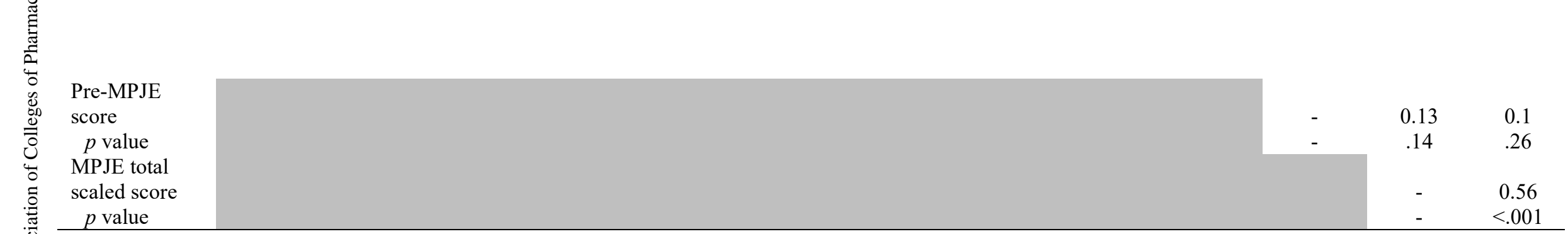

GPA, grade point average; MPJE, Multistate Pharmacy Jurisprudence Examination; PCAT, Pharmacy College Admission Test; PCOA, Pharmacy Curriculum Outcomes Assessment

${ }^{a}$ The four PCOA content areas are basic biomedical sciences (Area 1), pharmaceutical sciences (Area 2), social/behavioral/administrative sciences (Area 3), and clinical sciences (Area 4).

${ }^{b}$ MPJE pass/fail coded as $0=$ fail, $1=$ pass. In the case of MPJE pass/fail, the correlation is point biserial, rather than Spearman rho. 
Table 3. Multiple Linear Regression Model to Determine Value of Demographics, Pre-pharmacy Performance Indicators, and Pharmacy School Performance Indicators (Including PCOA Total Scaled Score) as Predictors of MPJE Total Scaled Score in the Class of $2020(\mathrm{n}=136)$

\begin{tabular}{|c|c|c|c|c|c|c|c|c|c|c|}
\hline & B & $\begin{array}{c}\text { Standard } \\
\text { Error }\end{array}$ & $\begin{array}{c}\text { Standardized } \\
\text { Beta }\end{array}$ & $t$ & $\begin{array}{c}p \\
\text { value }\end{array}$ & $\begin{array}{l}\text { Zero-order } \\
\text { Correlation }\end{array}$ & $\begin{array}{c}\text { Partial } \\
\text { Correlation }\end{array}$ & $\begin{array}{c}\text { Part } \\
\text { Correlation }\end{array}$ & Tolerance $^{a}$ & VIF $^{\mathbf{a}}$ \\
\hline Constant & 72.8 & 7.56 & & 9.63 & $<.001$ & & & & & \\
\hline Age at graduation & -0.09 & 0.08 & -0.09 & -1.11 & .27 & -0.18 & -0.1 & -0.08 & 0.91 & 1.1 \\
\hline Gender $^{\mathrm{a}}$ & -0.58 & 0.62 & -0.08 & -0.94 & .35 & -0.02 & -0.09 & -0.07 & 0.83 & 1.21 \\
\hline $\begin{array}{l}\text { Race/ethnicity } \\
\text { PCAT Composite }\end{array}$ & -0.21 & 0.7 & -0.02 & -0.31 & .76 & -0.16 & -0.03 & -0.02 & 0.89 & 1.12 \\
\hline Score & -0.02 & 0.02 & -0.08 & -0.9 & .74 & 0.12 & -0.08 & -0.07 & 0.66 & 1.52 \\
\hline $\begin{array}{l}\text { Pre-pharmacy GPA } \\
\text { Pharmacy law course }\end{array}$ & -1.06 & 0.88 & -0.1 & -1.2 & .23 & 0.2 & -0.12 & -0.09 & 0.63 & 1.58 \\
\hline $\begin{array}{l}\text { Final grade } \\
\text { Academic difficulty } \\
\text { during PharmD }\end{array}$ & 2.81 & 0.72 & 0.51 & 3.93 & $<.001$ & 0.53 & 0.34 & 0.29 & 0.33 & 3.07 \\
\hline program $^{\mathrm{a}}$ & 0.16 & 0.99 & 0.02 & 0.16 & .88 & -0.21 & 0.01 & 0.01 & 0.57 & 1.75 \\
\hline $\begin{array}{l}\text { Final pharmacy GPA } \\
\text { PCOA total scaled }\end{array}$ & -0.59 & 2.11 & -0.05 & -0.28 & .78 & 0.43 & -0.03 & -0.02 & 0.19 & 5.2 \\
\hline score & 0.03 & 0.01 & 0.29 & 2.61 & .01 & 0.4 & 0.23 & 0.19 & 0.45 & 2.22 \\
\hline Pre-MPJE score & 0.03 & 0.06 & -0.02 & -0.2 & .84 & 0.17 & -0.02 & -0.02 & 0.83 & 1.21 \\
\hline
\end{tabular}

aTolerance less than 0.1 and VIF greater than 10 indicate multicollinearity.

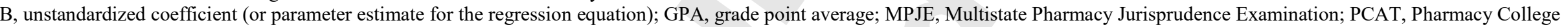
Admission Test; PCOA, Pharmacy Curriculum Outcomes Assessment; $t$, t-value used in hypothesis testing; VIF, variance inflation factor

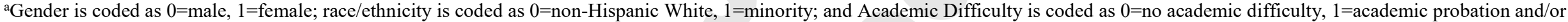
appearance before the Academic Standing and Promotion Review Committee. 
Table 4. Multiple Linear Regression Model to Determine Value of Demographics, Pre-pharmacy Performance Indicators, and Pharmacy School Performance Indicators (including PCOA Content Area Scores) as Predictors of MPJE Total Scaled Score in the Class of 2020 ( $\mathrm{n}=136)$

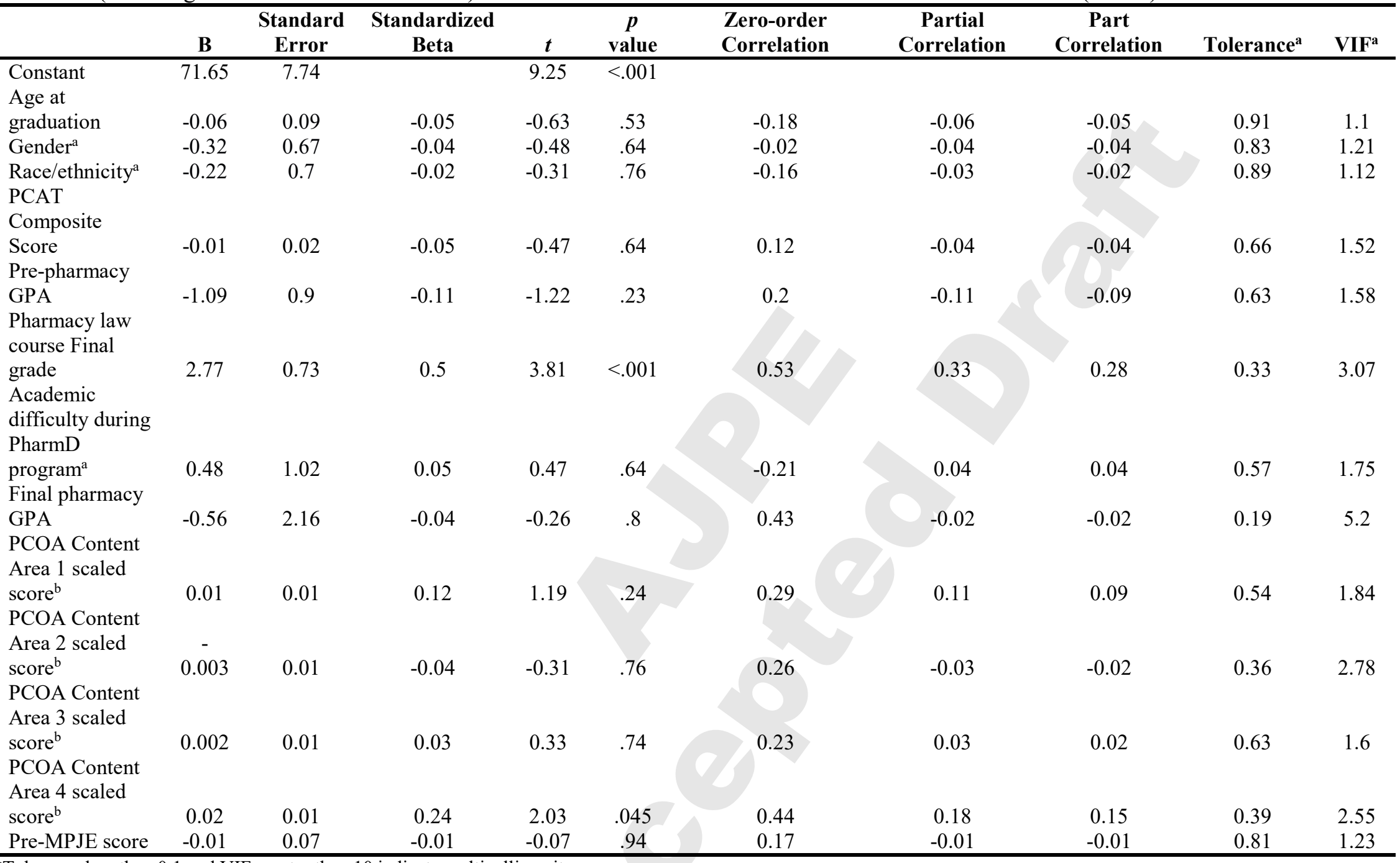

Tolerance less than 0.1 and VIF greater than 10 indicate multicollinearity.

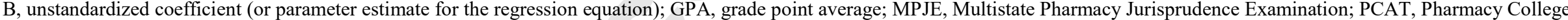
Admission Test; PCOA, Pharmacy Curriculum Outcomes Assessment; $t$, t-value used in hypothesis testing; VIF, variance inflation factor

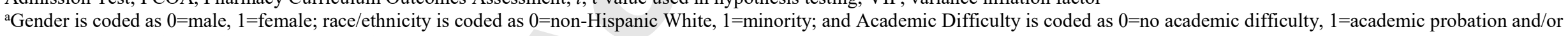
appearance before the Academic Standing and Promotion Review Committee.

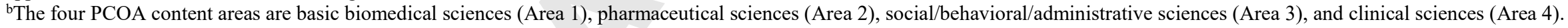


Table 5. Multiple Linear Regression Models to Determine Value of Demographics, Pre-pharmacy Performance Indicators, and Pharmacy School Performance Indicators as Predictors of MPJE Total Scaled Score in Two Subgroups of the Class of 2020, Those Who Took the Tennessee MPJE ( $\mathrm{n}=101$ ) and Those Who Took an Out-of-State MPJE $(\mathrm{n}=35)$

\begin{tabular}{|c|c|c|c|c|c|c|c|c|c|c|}
\hline & B & $\begin{array}{c}\text { Standard } \\
\text { Error }\end{array}$ & $\begin{array}{c}\text { Standardized } \\
\text { Beta }\end{array}$ & $t$ & $\begin{array}{c}p \\
\text { value }\end{array}$ & $\begin{array}{l}\text { Zero-order } \\
\text { Correlation }\end{array}$ & $\begin{array}{c}\text { Partial } \\
\text { Correlation }\end{array}$ & $\begin{array}{c}\text { Part } \\
\text { Correlation }\end{array}$ & Tolerance $^{a}$ & VIF $^{a}$ \\
\hline \multicolumn{11}{|l|}{ Tennessee (In-state) } \\
\hline \multicolumn{11}{|l|}{ MPJE } \\
\hline Constant & 70.2 & 8.39 & & 8.37 & $<.001$ & & & & & \\
\hline Age at graduation & -0.11 & 0.09 & -0.11 & -1.21 & .23 & -0.24 & -0.13 & -0.1 & 0.9 & 1.11 \\
\hline Gender $^{\mathrm{a}}$ & -0.2 & 0.72 & -0.03 & -0.28 & .78 & 0.09 & -0.03 & -0.02 & 0.76 & 1.32 \\
\hline Race/ethnicity ${ }^{\mathrm{a}}$ & -0.46 & 0.82 & -0.05 & -0.56 & .58 & -0.19 & -0.06 & -0.05 & 0.84 & 1.19 \\
\hline PCAT composite score & -0.01 & 0.02 & -0.06 & -0.58 & .57 & 0.09 & -0.06 & -0.05 & 0.64 & 1.56 \\
\hline Pre-pharmacy GPA & -0.83 & 0.96 & -0.09 & -0.87 & .39 & 0.23 & -0.09 & -0.07 & 0.63 & 1.59 \\
\hline \multicolumn{11}{|l|}{ Pharmacy law course } \\
\hline Final grade & 2.45 & 0.82 & 0.46 & 2.99 & .004 & 0.58 & 0.3 & 0.25 & 0.3 & 3.4 \\
\hline $\begin{array}{l}\text { Academic difficulty } \\
\text { during PharmD program }{ }^{\text {a }}\end{array}$ & 0.3 & 1.18 & 0.03 & 0.26 & .8 & -0.27 & 0.03 & 0.02 & 0.48 & 2.08 \\
\hline Final pharmacy GPA & 1.35 & 2.44 & 0.12 & 0.56 & .58 & 0.51 & 0.06 & 0.05 & 0.16 & 6.14 \\
\hline PCOA total scaled score & 0.01 & 0.01 & 0.12 & 0.94 & .35 & 0.37 & 0.1 & 0.08 & 0.41 & 2.42 \\
\hline Pre-MPJE score & 0.01 & 0.08 & 0.01 & 0.11 & .92 & 0.18 & 0.01 & 0.01 & 0.81 & 1.24 \\
\hline \multicolumn{11}{|l|}{ Out-of-State MPJE } \\
\hline Constant & 82.74 & 19.28 & & 4.29 & $<.001$ & & & & & \\
\hline Age at graduation & -0.15 & 0.31 & -0.09 & -0.48 & .64 & 0.02 & -0.1 & -0.07 & 0.55 & 1.84 \\
\hline Gender ${ }^{\mathrm{a}}$ & -1.6 & 1.53 & -0.19 & -1.05 & .31 & -0.27 & -0.21 & -0.15 & 0.6 & 1.66 \\
\hline Race/ethnicity ${ }^{\mathrm{a}}$ & 0.27 & 1.56 & 0.03 & 0.18 & .86 & -0.1 & 0.04 & 0.03 & 0.69 & 1.46 \\
\hline PCAT composite score & -0.02 & 0.04 & -0.09 & -0.48 & .63 & 0.19 & -0.1 & -0.07 & 0.58 & 1.72 \\
\hline Pre-pharmacy GPA & -2.54 & 2.42 & -0.23 & -1.05 & .31 & 0.09 & -0.21 & -0.15 & 0.44 & 2.26 \\
\hline \multicolumn{11}{|l|}{ Pharmacy law course } \\
\hline Final grade & 4.81 & 1.76 & 0.71 & 2.74 & .01 & 0.42 & 0.5 & 0.39 & 0.31 & 3.25 \\
\hline Academic difficulty & & & & & & & & & & \\
\hline during PharmD program ${ }^{a}$ & -0.19 & 2.6 & -0.02 & -0.07 & .94 & -0.06 & -0.02 & -0.01 & 0.44 & 2.43 \\
\hline Final pharmacy GPA & -6.91 & 5.05 & -0.43 & -1.37 & .18 & 0.2 & -0.28 & -0.2 & 0.21 & 4.72 \\
\hline PCOA total scaled score & 0.07 & 0.03 & 0.62 & 2.71 & .01 & 0.53 & 0.49 & 0.39 & 0.4 & 2.5 \\
\hline Pre-MPJE score & -0.08 & 0.16 & -0.1 & -0.49 & .63 & 0.17 & -0.1 & -0.07 & 0.54 & 1.85 \\
\hline
\end{tabular}

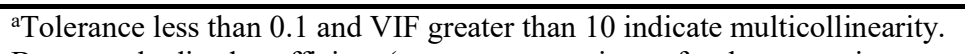

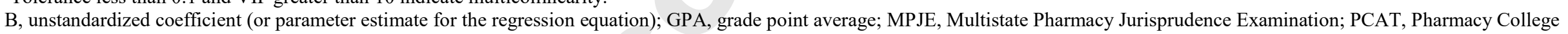
Admission Test; PCOA, Pharmacy Curriculum Outcomes Assessment; $t$, t-value used in hypothesis testing; VIF, variance inflation factor

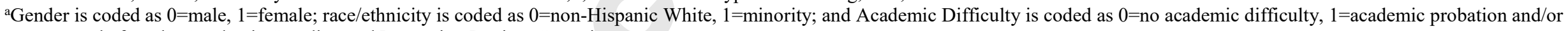
appearance before the Academic Standing and Promotion Review Committee 